\title{
MULTI-FUNCTION ACTIVE-ONLY HIGH-ORDER CURRENT-DRIVEN FILTER
}

\author{
MUHAMMAD TAHER ABUELMA'ATTI* \\ and HUSAIN ABDULLAH ALZAHER \\ King Fahd University of Petroleum and Minerals, Box 203, \\ Dhahran 31261, Saudi Arabia
}

(Received 29 April 2000; In final form 12 July 2000)

\begin{abstract}
A new multi-function high-order current-driven filter is proposed. The filter uses only operational amplifiers, and operational transconductance amplifiers (OTAs). Without using any external passive elements, a variety of high-order input-current/output-current and/or input-current/output-voltage responses can be realised without changing the circuit topology and without any matching or cancellation conditions. The parameters of the high-order filter responses can be electronically tuned by adjusting the bias currents of the OTAs.
\end{abstract}

Keywords: Active filters; Operational amplifiers; Operational transconductance amplifiers

\section{INTRODUCTION}

At present, there is a growing interest in designing capacitorlessresistorless current-mode active-only filters using only active-elements, such as operational amplifiers (OAs) and operational transconductance amplifiers (OTAs). This is attributed to their integratability, programmability and wide frequency range of operation $[1,2]$. The circuits reported in $[1,2]$ can realise second-order lowpass, highpass, bandpass and notch transfer functions, without any matching or cancellation conditions. Realisation of allpass transfer functions is also feasible.

*Corresponding author. 
The work to be presented in this paper is an attempt to extend the applications of capacitorless-resistorless active-only realisations to the domain of high-order filters. Obviously, high-order filters can be obtained by cascading second-order realisations. However, this approach usually requires an extensive number of active components. For example, the realisation of an $N$ th-order response, $N=$ even integer, using the structure proposed in [2] requires $N$ OAs and $5 N / 2$ OTAs. Similarly, the realisation of an $N$ th-order response using the structure proposed in [1] requires $3 N / 2$ OTAs and $N$ OAs. It will be shown, in this paper, that an $N$ th-order response, with $N=$ even integer, can be realised using $N$ OAs and $N+1$ OTAs only. Thus, the proposed realisation saves $(N / 2-1)$ OTAs. Moreover, while the realisations of [1] and [2] can support only one output response at a time, the proposed realisation can simultaneously support a variety of output responses.

\section{PROPOSED CIRCUIT}

The proposed circuit is shown in Figure 1. Using standard notations, the OTA can be characterised by $i=g_{m_{k}}\left(v_{+}-v_{-}\right)$, where $g_{m_{k}}=$ $\left(I_{A B C k} / 2 V_{T}\right)$ is the transconductance of the $k$ th OTA, $I_{A B C k}$ is the auxiliary bias-current, $V_{T}$ is the thermal voltage and $v_{+}$and $v_{-}$are

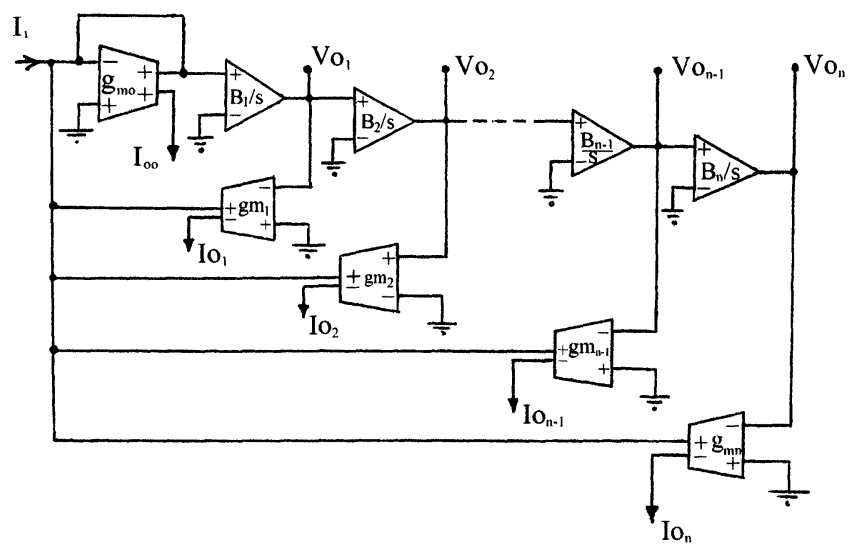

FIGURE 1 Proposed active-only $n$ th-order filter. 
the input voltages of the OTA. Assuming internally compensated operational amplifiers with open-loop gain of $A=\left(B_{k} / s\right)$, where $B_{k}$ is the gain-bandwidth product of the $k$ th operational amplifier, routine analysis yields the current transfer functions given by

$$
\begin{aligned}
\frac{I_{o_{k}}}{I_{i}} & =\frac{\left(g_{m_{k}} / g_{m_{o}}\right) \prod_{m=1}^{k} B_{m} s^{n-k}}{s^{n}+\left(g_{m_{1}} / g_{m_{o}}\right) B_{1} s^{n-1}+\left(g_{m_{2}} / g_{m_{o}}\right) B_{1} B_{2} s^{n-2}+\cdots+\left(g_{m_{n}} / g_{m_{o}}\right) \prod_{m=1}^{n} B_{m}} \\
& =\frac{a_{k} s^{n-k}}{s^{n}+b_{1} s^{n-1}+b_{2} s^{n-2}+\cdots+b_{n}}
\end{aligned}
$$

and the voltage transfer functions given by

$$
\begin{aligned}
\frac{V_{o_{k}}}{I_{i}} & =\frac{\left(1 / g_{m_{o}}\right) \prod_{m=1}^{k} B_{m} s^{n-k}}{s^{n}+\left(g_{m_{1}} / g_{m_{o}}\right) B_{1} s^{n-1}+\left(g_{m_{2}} / g_{m_{o}}\right) B_{1} B_{2} s^{n-2}+\cdots+\left(g_{m_{n}} / g_{m_{o}}\right) \prod_{m=1}^{n} B_{m}} \\
& =\frac{c_{k} s^{n-k}}{s^{n}+b_{1} s^{n-1}+b_{2} s^{n-2}+\cdots+b_{n}}
\end{aligned}
$$

Thus, the circuit of Figure 1 can, simultaneously, support $(n+1)$ highimpedance output currents and $n$ low-impedance output voltages.

From (1) and (2) the parameters $a_{k}, k=0-n$ and $c_{k}, k=1-n$ can be expressed as

$$
a_{k}=\frac{g_{m_{k}}}{g_{m_{o}}} \prod_{m=1}^{k} B_{m}
$$

and

$$
c_{k}=\frac{a_{k}}{g_{m_{k}}}
$$

And the parameters $b_{l}, l=1-n$ can be expressed as

$$
b_{l}=\frac{g_{m_{l}}}{g_{m_{o}}} \prod_{m=1}^{l} B_{m}
$$

From (5) it can be seen that the parameters $b_{l}, l=1-n$ can be independently adjusted by controlling the transconductances $g_{m_{l}}$, $l=1-n$. Thus, the proposed circuit enjoys electronic tunability of its parameters. 


\section{SIMULATION RESULTS}

The circuit of Figure 1 was used to realise the 4th-order lowpass Butterworth current transfer function given by (Tab. 2.2 of [3])

$$
H(s)=\frac{1}{s^{4}+2.61312593 s^{3}+3.41421356 s^{2}+2.61312593 s+1}
$$

Equation (6) can be written in a normalised form as follows

$$
\begin{aligned}
& H\left(\frac{s}{\omega_{o}}\right) \\
& \quad=\frac{\omega_{o}^{4}}{s^{4}+2.61312593 \omega_{o} s^{3}+3.41421356 \omega_{o}^{2} s^{2}+2.61312593 \omega_{o}^{3} s+\omega_{o}^{4}}
\end{aligned}
$$

With $\omega_{o}=2 \pi \mathrm{Mrad} / \mathrm{sec}, B=2 \pi(4.1345) \mathrm{Mrad} / \mathrm{sec}$ and if we choose $g_{m_{o}}=200 \mu \mathrm{A} / \mathrm{V}$ then $g_{m_{1}}=126.41 \mu \mathrm{A} / \mathrm{V}, g_{m_{2}}=39.95 \mu \mathrm{A} / \mathrm{V}, g_{m_{3}}=$ $7.395 \mu \mathrm{A} / \mathrm{V}, g_{m_{4}}=0.684 \mu \mathrm{A} / \mathrm{V}$. The operational amplifier LF156, with $B=2 \pi(4.1345) \mathrm{Mrad} / \mathrm{sec}$ and the OTA macromodel [4] were used in simulation. With a dc supply $\pm 10 \mathrm{~V}$, the results obtained are shown in Figure 2. It appears from Figure 2 that the agreement between simulated and theoretical results is good.

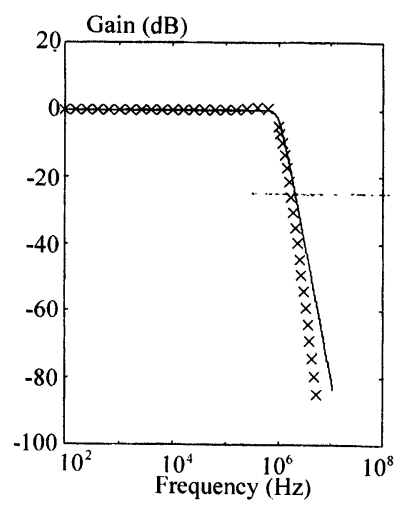

FIGURE 2 Simulated $(-)$ and calculated $(x)$ results obtained from the 4th-order lowpass response of (7). 


\section{CONCLUSION}

A new $N$ th-order current-driven filter has been presented. The proposed filter uses only operational amplifiers and operational transconductance amplifiers. No external passive elements are used. The proposed circuit enjoys the following advantages:

(a) Independent current control of the filter parameters.

(b) Simultaneous support of $n+1$ different high-impedance outputcurrent responses and $n$ different low-impedance output-voltage responses.

(c) Insensitivity to the temperature variations of the transconductances of the OTAs. Moreover, using operational amplifiers with temperature compensated gain-bandwidth products [5], highorder responses with temperature-insensitive parameters can be realised.

\section{References}

[1] Tsukutani, T., Ishida, M., Tsuiki, S. and Fukui, Y. (1996). Current-mode biquad without external passive elements, Electronics Letters, 32, 197-198.

[2] Abuelma'atti, M. T. and Alzaher, H. A. (1997). Universal three input and one output current-mode filter without external passive elements, Electronics Letters, 33, $281-283$.

[3] Chen, W.-K., Passive and Active Filters, John Wiley \& Sons, N.Y., 1986.

[4] Wu, J. (1994). Current-mode high-order OTA-C filters, International Journal of Electronics, 76, 1115-1120.

[5] Acar, C. and Ghausi, M. S. (1978). Design of high-order active filters in integrated circuits, IEEE Journal of Solid-State Circuits, SC-13, 273-276. 

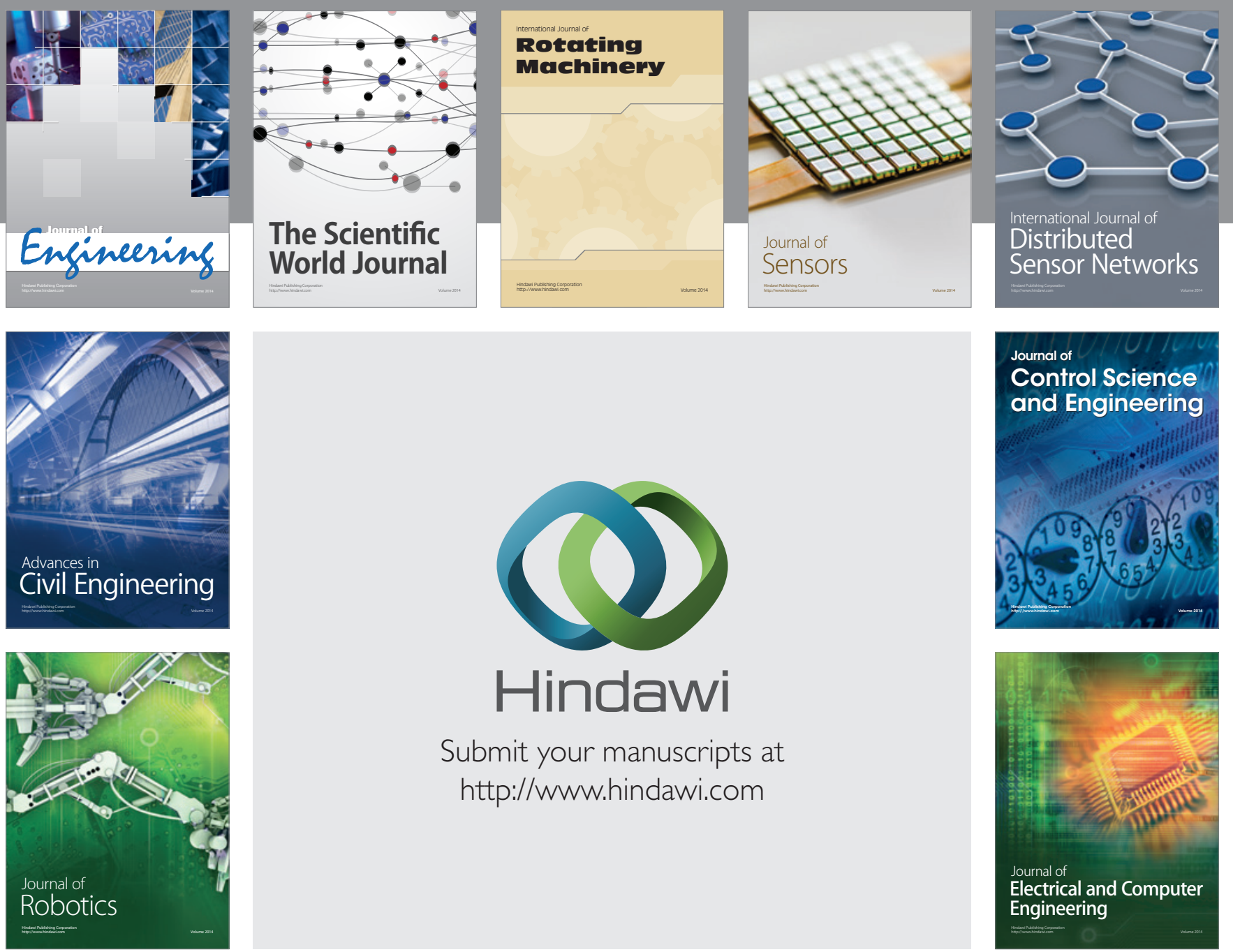

Submit your manuscripts at

http://www.hindawi.com
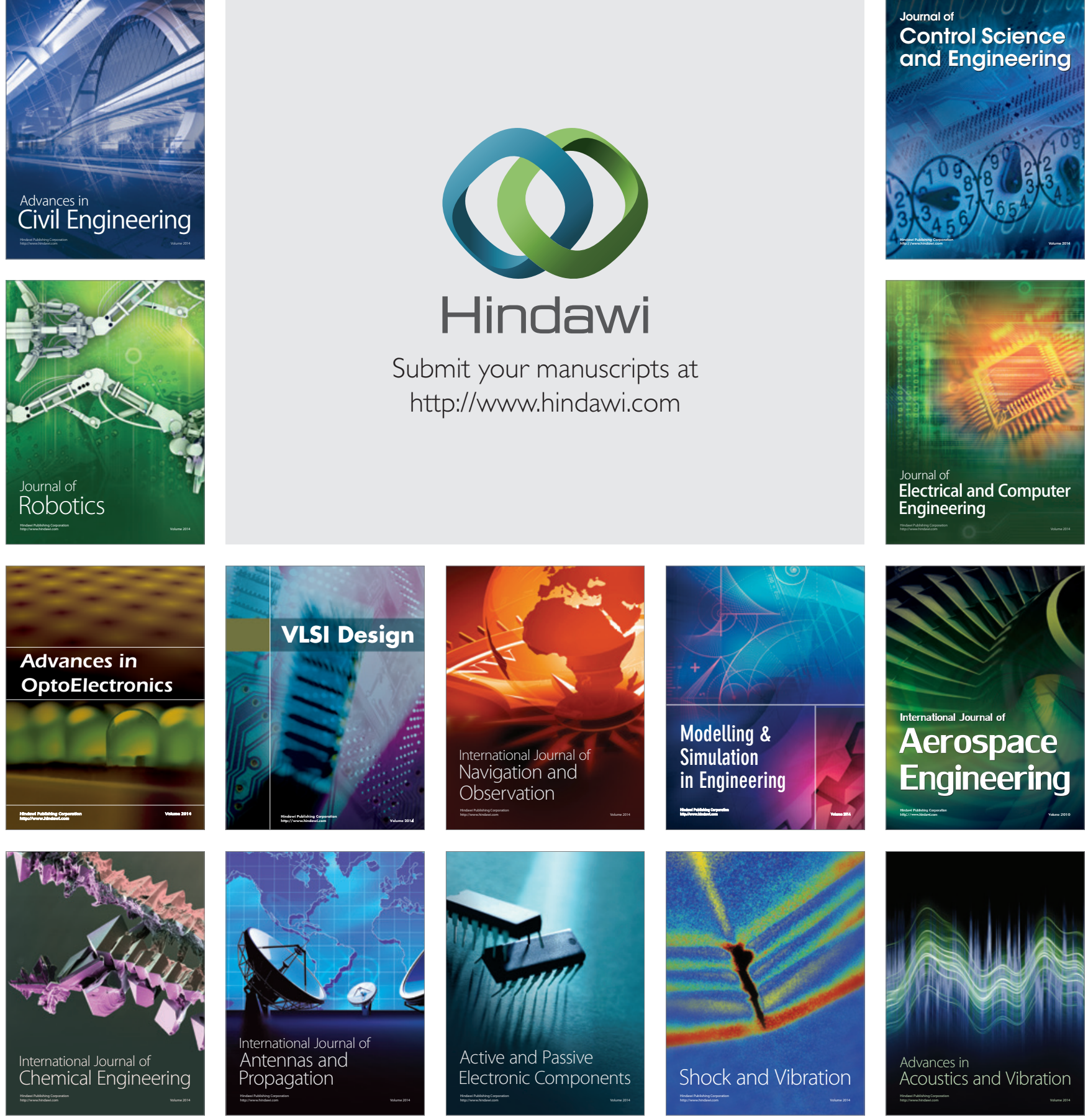